University of Nebraska - Lincoln

DigitalCommons@University of Nebraska - Lincoln

2-1-1999

\title{
Effect of uniform electric field on homogeneous vapor-liquid nucleation and phase equilibria. II. Extended simple point charge model water
}

\author{
G.T. Gao \\ University of Nebraska-Lincoln \\ K.J. Oh \\ University of Nebraska-Lincoln \\ Xiao Cheng Zeng \\ University of Nebraska-Lincoln, xzeng1@unl.edu
}

Follow this and additional works at: https://digitalcommons.unl.edu/chemzeng

Part of the Chemistry Commons

Gao, G.T.; Oh, K.J.; and Zeng, Xiao Cheng, "Effect of uniform electric field on homogeneous vapor-liquid nucleation and phase equilibria. II. Extended simple point charge model water" (1999). Xiao Cheng Zeng Publications. 63.

https://digitalcommons.unl.edu/chemzeng/63

This Article is brought to you for free and open access by the Published Research - Department of Chemistry at DigitalCommons@University of Nebraska - Lincoln. It has been accepted for inclusion in Xiao Cheng Zeng Publications by an authorized administrator of DigitalCommons@University of Nebraska - Lincoln. 


\title{
Effect of uniform electric field on homogeneous vapor-liquid nucleation and phase equilibria. II. Extended simple point charge model water
}

\author{
G. T. Gao, K. J. Oh, and X. C. Zeng ${ }^{\text {a) }}$ \\ Department of Chemistry, University of Nebraska-Lincoln, Lincoln, Nebraska 68588
}

(Received 24 August 1998; accepted 27 October 1998)

\begin{abstract}
The effects of a uniform electric field on homogeneous vapor-liquid nucleation of the extended simple point charge (SPC/E) model water have been simulated. A grand canonical Monte Carlo simulation approach [I. Kusaka et al., J. Chem. Phys. 108, 3416 (1998)], which directly gives the equilibrium distribution of physical clusters, is employed to calculate the formation free energy of the SPC/E water cluster. The results show that the formation free energy is lowered in a uniform field if the chemical potential of the supersaturated vapor is fixed; in this case, the field enhances the rate of nucleation. However, if the vapor supersaturation or pressure is fixed, the formation free energy increases with the field, that is, the field reduces the rate of nucleation. This conclusion is consistent with an earlier study using the $\mathrm{i} / \mathrm{v}$ cluster theory for weakly dipolar Stockmayer fluid in a uniform field (Part I). A byproduct of this work is the vapor-liquid coexistence (binodal) of the $\mathrm{SPC} / \mathrm{E}$ water in the presence of the electric field. (C) 1999 American Institute of Physics. [S0021-9606(99)50605-3]
\end{abstract}

\section{INTRODUCTION}

For a supersaturated dipolar vapor in a uniform electric field, an open question is whether the field enhances or reduces the rate of vapor-liquid nucleation. To date, most experimental studies have focused on the effects of the electric field on nucleation of fluids containing ions. The effects are inferred from measurements using various types of cloud chambers. Rabeony and Mirabel ${ }^{1}$ conducted the first study of the field effects on nucleation. They found that the electric field tends to lower the nucleation rate first; then the rate levels off at a higher field with strength $E>1500 \mathrm{~V} / \mathrm{m}$. Later, $\mathrm{He}$ and Hopke ${ }^{2}$ discovered that the rate can actually increase considerably in an electric field. They attributed the large increase to the lessened vapor depletion by the field, which leads to a more efficient ion-induced nucleation. In another recent experiment, Katz et al. ${ }^{3}$ found that the nucleation rate is essentially proportional to the ion density but independent of the strength of the field if the strength is less than $10^{4} \mathrm{~V} / \mathrm{m}$. An important result of Katz et al.'s experiment is that a much stronger field is needed to see appreciable field effects on nucleation, particularly for fluids without containing ions.

On the theoretical side, most previous studies were based on the classical nucleation theory. ${ }^{4}$ Kashchiev ${ }^{5}$ derived a formula for the formation free energy of liquid clusters and showed that if the dielectric permeability of the clusters is smaller than that of the supersaturated vapor, the electric field will stimulate the cluster formation, while in the opposite situation it will inhibit this process. Isard, ${ }^{6}$ taking into account the effect of the boundary, found that the formation free energy is always lowered in a uniform electric field, even when the dielectric permeability is larger than that of the supersaturated vapor. Cheng, ${ }^{7}$ assuming the droplet to

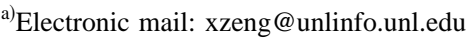

have a prolate spheroid shape, showed that an electric field increases the probability of droplet nucleation. Recently, Warshavsky and Shchekin ${ }^{8}$ demonstrated, by taking into account the elongation of the droplet in the field, that the electric field enhances the nucleation rate by decreasing the chemical potential of the droplet.

To understand the nucleation in the field from a molecular level, we have employed a molecular theory of nucleation-the $\mathrm{i} / \mathrm{v}$ cluster theory developed by Reiss and co-workers $^{9-13}$ to study a weakly dipolar system, the Stockmayer fluid with a reduced dipole moment $\mu^{*}=\sqrt{2}$. Results of this work were published in Ref. 14 (hereafter called part I). The major findings of part I were that the field can either enhance or reduce the nucleation rate depending upon the state of the supersaturated vapor. If the supersaturation of the vapor is fixed, the field will raise the formation free energy and thus reduce the nucleation rate. However, if the chemical potential of the vapor is fixed (which may be difficult to achieve experimentally) the field will reduce the formation free energy and thus enhance the nucleation rate.

However, the $\mathrm{i} / \mathrm{v}$ cluster theory has one apparent disadvantage: the formation free energy can only be inferred qualitatively but not quantitatively, because of the underlying decoupling approximation. Moreover, the theory requires the input of the Helmholtz free energy of the cluster, ${ }^{11,13}$ which is usually obtained via thermodynamic integration of the equation of state. ${ }^{15}$ Consequently, extensive computer simulation $^{15}$ is needed to achieve a smooth curve of the integrand. ${ }^{16}$ This computational task is particularly arduous for strongly dipolar system such as extended simple point charge $(\mathrm{SPC} / \mathrm{E})$ water (having a reduced dipole moment $\left.\mu^{*}=4.01\right){ }^{17}$ A test calculation by us indicated that the use of $10^{8}$ Monte Carlo steps per particle is still insufficient to obtain a smooth curve of the integrand.

Recently Kusaka, Wang, and Seinfeld ${ }^{18}$ developed a new 
approach to calculate formation free energy for nonvolatile fluids. The word nonvolatile here means fluid having extremely low vapor pressures. Strongly dipolar fluids such as SPC/E water belong to this class. The essence of this approach is to determine the equilibrium cluster size distribution via grand canonical Monte Carlo (GCMC) simulation. From this distribution, the reversible work of formation (or formation free energy) of a cluster in the vapor phase can be obtained. Compared with the $\mathrm{i} / \mathrm{v}$ cluster method, this approach is computationally less demanding and yet can yield a formation free energy quantitatively. However, the extension of this approach for weakly dipolar or nonpolar systems such as Lennard-Jones fluid is still a challenge.

In this work, we employed the GCMC simulation method of Kusaka et al. to study the field effects on nucleation of SPC/E model water. To achieve the condition of fixed supersaturation of the vapor, the equilibrium vapor pressure data with and without the presence of the field are needed. To this end, the vapor-liquid phase coexistence curves (binodals) of the SPC/E water were obtained using the Gibbs ensemble Monte Carlo (GEMC) simulation. ${ }^{19,20}$

Despite some obvious shortcomings ${ }^{21}$ simple pair potentials for water such as SPC, ${ }^{22} \mathrm{SPC} / \mathrm{E},{ }^{17}$ and TIP4P ${ }^{23}$ are quite successful for reproducing many thermophysical properties of water under the ambient condition. In these models, many-body polarization contributions are treated via an effective permanent dipole moment larger than the value in the gas phase. The SPC/E model is chosen here in particular because it yields an excellent fit to the vapor-liquid coexistence (binodal) of laboratory water. ${ }^{24,25}$

This paper is organized as follows. In Sec. II, we present the results of GEMC simulation of the SPC/E water in the presence of a uniform electric field (including zero field). In Sec. III, we report the GCMC simulation results of the formation free energy from which we examine the field effects on the homogeneous vapor-liquid nucleation. A discussion and concluding remarks will be presented in Sec. IV.

\section{VAPOR-LIQUID COEXISTENCE OF SPC/E WATER IN ELECTRIC FIELD}

The SPC/E water molecule consists of three point charges located at the three sites representing one oxygen and two hydrogens, respectively. The $\mathrm{OH}$ bond length is set to be $1.0 \AA$ and the $\mathrm{HOH}$ bond angle is defined as $109.47^{\circ}$. Besides the charge-charge interactions, there is also a Lennard-Jones interaction between each pair of oxygens. The full interaction potential between two molecules is written as:

$$
u\left(r_{i j}\right)=4 \epsilon\left[\left(\frac{\sigma_{o o}}{r_{o o}}\right)^{12}-\left(\frac{\sigma_{o o}}{r_{o o}}\right)^{6}\right]+\sum_{i=1}^{3} \sum_{j=1}^{3} \frac{q_{i} q_{j}}{r_{i j}}
$$

where $\sigma_{o o}=3.167 \AA, \epsilon / k_{B}=78.21 \mathrm{~K}$, the charge for the hydrogen site $q_{\mathrm{H}}=0.4238 e$, and the charge for the oxygen site $q_{\mathrm{O}}=-2 q_{\mathrm{H}}$. Here $k_{B}$ is the Boltzmann constant and $e$ is the elementary charge of the electron. In the absence of the electric field, the total potential energy is given by
TABLE I. GEMC simulation results of the equilibrium vapor and liquid coexistence densities of the SPC/E water under the electric field $E=0,1.0$ $\times 10^{9} \mathrm{~V} / \mathrm{m}$, and $2.0 \times 10^{9} \mathrm{~V} / \mathrm{m}$. The unit of density is in $\mathrm{kg} \mathrm{m}^{-3}$.

\begin{tabular}{|c|c|c|c|c|c|c|}
\hline \multirow[b]{2}{*}{$T(\mathrm{~K})$} & \multicolumn{2}{|c|}{$E=0$} & \multicolumn{2}{|c|}{$E=1.0 \times 10^{9} \mathrm{~V} / \mathrm{m}$} & \multicolumn{2}{|c|}{$E=2.0 \times 10^{9} \mathrm{~V} / \mathrm{m}$} \\
\hline & $\rho_{L}$ & $\rho_{G}$ & $\rho_{L}$ & $\rho_{G}$ & $\rho_{L}$ & $\rho_{G}$ \\
\hline 298.15 & 1012.4 & 0.007 & $\ldots$ & $\ldots$ & $\ldots$ & $\ldots$ \\
\hline 323 & 976.6 & 0.026 & 996.4 & 0.019 & $\cdots$ & $\cdots$ \\
\hline 373 & 935.2 & 0.238 & 952.2 & 0.157 & $\cdots$ & $\cdots$ \\
\hline 423 & 903.4 & 1.57 & 913.3 & 0.903 & $\ldots$ & $\ldots$ \\
\hline 473 & 831.1 & 4.82 & 852.7 & 3.30 & 865.0 & 2.40 \\
\hline 523 & 765.3 & 16.4 & 780.9 & 10.6 & 802.6 & 6.72 \\
\hline 573 & 647.2 & 48.8 & 675.3 & 25.7 & 723.0 & 17.0 \\
\hline 600 & 466.3 & 60.9 & 577.1 & 35.5 & 652.1 & 27.4 \\
\hline 623 & $\cdots$ & $\cdots$ & 506.7 & 84.8 & 610.9 & 42.3 \\
\hline 650 & $\cdots$ & $\cdots$ & $\cdots$ & $\cdots$ & 495.7 & 74.6 \\
\hline
\end{tabular}

$$
U_{0}=\sum_{i} \sum_{i<j} u\left(r_{i j}\right)
$$

in a uniform electric field $\mathbf{E}$, the total potential energy becomes:

$$
U=U_{0}-\mathbf{M} \cdot \mathbf{E},
$$

where $\mathbf{M}$ is the sum of the molecular dipole moments.

The GEMC method of Panagiotopoulos ${ }^{19,20}$ is employed to obtain the vapor-liquid coexistence curves. In this method, two simulation cells are used, each representing a portion of one coexisting phase. Three types of Monte Carlo moves were taken in the simulation: molecular displacement and rotation to assure internal equilibrium; volume rearrangement of the cells to satisfy equal pressure conditions; and molecular exchange between two cells to enforce equal chemical potential conditions. For the Lennard-Jones part of the potential, spherical cutoff approximation with long range correction was taken. ${ }^{15}$ The cutoff distance was set to be half of the simulation cell. The long-ranged Coulombic interactions were handled by the Ewald sum under the 'tin-foil' boundary condition. ${ }^{26}$ All systems consist of 256 particles. Each Monte Carlo (MC) cycle consists of 256 attempts of displacement and rotation, one volume rearrangement attempt, and several hundreds of particle transfer attempts. A complete simulation includes 40000 MC cycles; 20000 cycles for equilibration, and the other 20000 cycles for data collection. At low temperatures, particularly near the triple point because the successful particle transfer rate becomes very low, one thousand particle transfer attempts in conjunction with $60000 \mathrm{MC}$ cycles for equilibration were used. All results of GEMC simulation are given in Table I.

We note that the vapor-liquid coexistence of the SPC/E water has been calculated by Guissani and Guilot, ${ }^{24}$ using the thermodynamics integration method and by Alejandre et $a l .{ }^{25}$ in the study of vapor-liquid interfaces through molecular dynamics simulation. Both reported that the SPC/E model can reproduce the vapor-liquid coexistence (binodal) of laboratory water very well.

In Fig. 1 we show the vapor-liquid coexistence curve of the SPC/E water from the GEMC simulation, and also that from the molecular dynamics (MD) simulation by Alejandre 


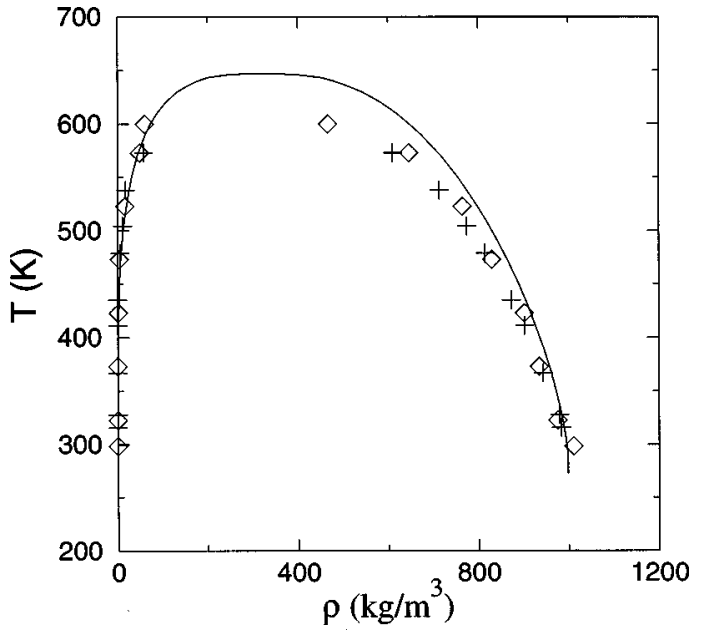

FIG. 1. Binodal curve of the SPC/E water in zero field. The solid line represents the experimental values (Ref. 27), the open diamonds are GEMC results of this work, and the crosses are MD results of Alejandre et al. (Ref. 25).

et $a l .{ }^{25}$ as well as the one from the experiment. ${ }^{27}$ One can see that the agreement between our results and those of Alejandre et al. is excellent. (Alejandre et al. have already shown that their results are in good agreement with those of Guissani and Guilot. ${ }^{24}$ ) From Fig. 1, one can also see good agreement between the simulation and the experiment over a wide range of temperatures. Note however that as shown in Table I, the equilibrium vapor densities of the SPC/E water at low temperatures are too low compared to the experimental values.

Table I also shows vapor-liquid coexistence data for SPC/E water in electric fields, for the field strength $E=1.0$ $\times 10^{9} \mathrm{~V} / \mathrm{m}$ and $E=2.0 \times 10^{9} \mathrm{~V} / \mathrm{m}$, respectively. The strength of the fields is comparable to the average value of the local internal electric field in condensed water. (Note that a field strength on the order of $10^{10} \mathrm{~V} / \mathrm{m}$ is within the operating range of the modern laser.) It was pointed out that any external electric field with strength much less than 5 $\times 10^{9} \mathrm{~V} / \mathrm{m}$ would have a negligible effect on the thermophysical properties of real water. ${ }^{21}$ For SPC/E water, we found that when $E=0.5 \times 10^{8} \mathrm{~V} / \mathrm{m}$ was used there are no noticeable changes in the binodal curve compared to the zero-field one.

In Fig. 2 three binodal curves are displayed. It can be seen that in the electric field the equilibrium vapor density becomes smaller and the liquid density becomes larger. Therefore, the critical temperature becomes higher. The stronger the applied field is, the greater the density changes. This is because the applied field effectively enhances the attractive interaction between dipolar molecules. These results are consistent with those of Stevens and $\mathrm{Grest}^{28}$ who studied the vapor-liquid coexistence of Stockmayer fluid in a uniform field.

\section{GRAND CANONICAL MONTE CARLO SIMULATION OF SPC/E WATER CLUSTER IN ELECTRIC FIELD}

Recently, Kusaka et al. ${ }^{18}$ devised a new approach in identifying the physical cluster. The latter is defined as the

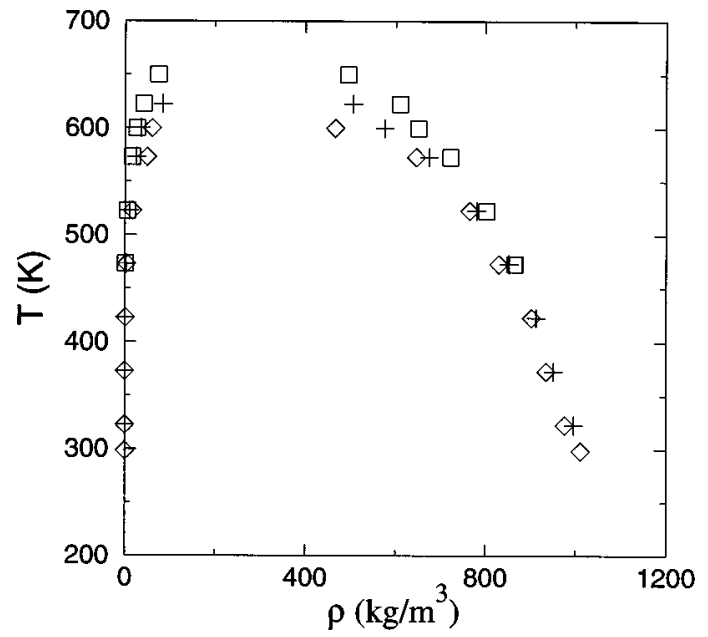

FIG. 2. Binodal curves of the SPC/E water under the field: $E=0$ (open diamonds), $E=1.0 \times 10^{9} \mathrm{~V} / \mathrm{m}$ (crosses), and $E=2.0 \times 10^{9} \mathrm{~V} / \mathrm{m}$ (open squares).

density fluctuation that leads to nucleation. This new approach is based on the observation that in typical nucleation measurements, since the temperature is usually well below the critical point, the large density fluctuations which can lead to condensation are far enough apart and that they may be regarded as almost mutually decoupled. The key idea in this approach is to select a Monte Carlo simulation cell with volume $V$ such that $V$ is large enough to allow one to describe the relevant nucleation process in a supersaturated vapor, and yet small enough to suppress the density fluctuations irrelevant to the nucleation event. For the latter requirement, Kusaka et al. chose $V$ to satisfy

$$
n_{v} V \ll 1
$$

where $n_{v}$ is the number density of the supersaturated vapor. For SPC/E model water at temperature $T=298.15 \mathrm{~K}$ and with supersaturated vapor density $\left(n_{v}=z \Omega\right)$ ranging from $0.08 \times 10^{-5} \AA^{-3}$ to $0.23 \times 10^{-5} \AA^{-3}$, they chose $V$ to be a spherical cavity with a radius of $15 \AA$. Here, $z=e^{\beta \mu} / \Lambda^{3}$ is the activity, $\Lambda$ is the de Broglie wavelength, $\mu$ is the chemical potential, and $\beta=\left(k_{B} T\right)^{-1} ; \Omega$ is the integral result of the rotational coordinate of a molecule in the ideal gas. Kusaka et al. argued that the cavity on average contains no vapor molecules and the number of molecules $N_{c}$ generated in the cell is in fact the size of the physical cluster.

Kusaka et al. then used an umbrella-sampling grand canonical MC method ${ }^{29}$ to calculate the equilibrium cluster size distribution $c\left(N_{c}\right)=p\left(N_{c}\right) / V$, defined as the probability of finding a physical cluster of size $N_{c}$ (or an $N_{c}$ cluster as called by Kusaka et al.) in a unit volume. Here, $p\left(N_{c}\right)$ is the probability of finding an $N_{c}$ cluster in the volume $V$ and is given by

$$
p\left(N_{c}\right)=\frac{\Xi_{c}\left(\beta, V, z ; N_{c}\right)}{\Xi(\beta, V, z)},
$$

where

$$
\Xi(\beta, V, z)=\sum_{N=0}^{N_{\text {cut }}} \frac{z^{N}}{N !} \int d\{N\} e^{-\beta U_{N}}
$$




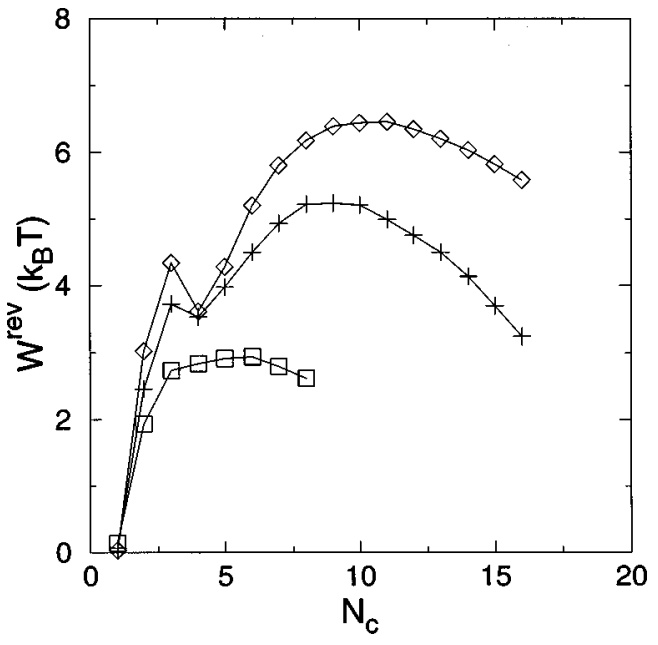

FIG. 3. The formation free energy of the SPC/E water cluster at $T$ $=298.15 \mathrm{~K}$ and $z \Omega=0.23 \times 10^{-5} \AA^{-3}$, and under the field: $E=0$ (open diamonds), $E=1.0 \times 10^{9} \mathrm{~V} / \mathrm{m}$ (crosses), and $E=1.5 \times 10^{9} \mathrm{~V} / \mathrm{m}$ (open squares).

is the grand partition function and

$$
\Xi_{c}\left(\beta, V, z ; N_{c}\right)=\frac{z^{N_{c}}}{N !} \int d\left\{N_{c}\right\} e^{-\beta U_{N_{c}}}
$$

is the term in the grand partition function $\Xi$ for $N=N_{c} ; U_{N}$ is the total potential energy for system with $N$ molecules; $\{N\}$ denotes the collection of all translational and rotational coordinates of the $N$ molecules; $N_{\text {cut }}$ is the upper bound of the molecular number that ensures the system in a metastable state. Once the cluster size distribution is determined, the reversible work of formation (or the formation free energy) for an $N_{c}$ cluster can be determined via

$$
W^{\mathrm{rev}}\left(N_{c}\right)=-\log \frac{c\left(N_{c}\right)}{n_{v}} .
$$

For more theoretical details, the reader is referred to the work of Kusaka et al. ${ }^{18}$

In this work, we applied Kusaka et al.'s method to study the nucleation of SPC/E water in a uniform electric field. We used the same value of radius of spherical cavity $(15 \AA)$ as in Ref. 18. Here, the only modification is the relationship between the vapor density $n_{v}$ and the product $z \Omega$. Note that for an ideal SPC/E vapor, $n_{v}=z \Omega$. In a uniform electric field, however, the vapor density is given by: ${ }^{30}$

$$
n_{v}=z \Omega f(x),
$$

and the pressure is given by

$$
P=z \Omega k_{B} T f(x),
$$

where

$$
f(x)=\frac{\sinh (x)}{x},
$$

and $x=\beta m E$, where $m$ is the value of the molecular dipole moment. For SPC/E water, $m=2.35 D$. The factor $f(x)$ in Eqs. (9) and (10) is resulted from the integration of the rotational coordinate under the influence of the field.

In Figs. 3 and 4 we showed the GCMC results of the

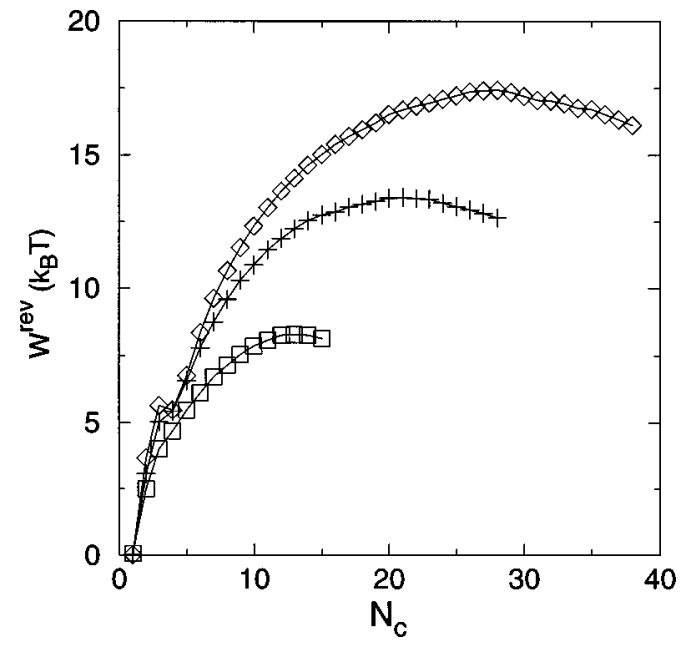

FIG. 4. The same as Fig. 3, but for $z \Omega=0.12 \times 10^{-5} \AA^{-3}$.

formation free energy versus the size of physical clusters. In Fig. 3, the vapor density $z \Omega=0.23 \times 10^{-5} \AA^{-3}$, and in Fig. $4, z \Omega=0.12 \times 10^{-5} \AA^{-3}$. The three curves in both Figs. 3 and 4 correspond to three different field strengths: $E=0$, $1.0 \times 10^{9} \mathrm{~V} / \mathrm{m}$, and $1.5 \times 10^{9} \mathrm{~V} / \mathrm{m}$. The temperature is always set at $298.15 \mathrm{~K}$ and the chemical potential is also fixed, both with and without the presence of the fields. Figures 3 and 4 show that in the electric field the formation free energy is lowered and thus the size of the critical cluster becomes smaller; the higher the field strength is, the lower the formation free energy and the smaller the critical cluster sizes. This behavior can be understood as follows. Because $f(x)>1$ for $x>0$, when the external electric field is applied, one can see from Eqs. (9) and (10) that the pressure of the supersaturated vapor will increase if the chemical potential is fixed. In addition, as shown in Sec. II, the equilibrium coexistence vapor density and the saturation pressure $P_{\text {sat }}$ decrease as the electric field increases. Therefore, the supersaturation $S$ $=P / P_{\text {sat }}$ of the supersaturated vapor increases, which results in a lower formation free energy and a smaller critical cluster size.

Besides the fixed chemical potential condition, we also examined the fixed supersaturation condition for the supersaturated vapor. In this case, since both the factor $f(x)$ and the saturation pressure $P_{\text {sat }}$ are a function of the field strength, to maintain a fixed supersaturation, the value of $z \Omega$ has to be adjusted whenever the field strength is changed. We performed a grand canonical simulation at fixed supersaturation $S$ and temperature $T=323 \mathrm{~K}$. For $E=0, z \Omega$ is set to be $0.5 \times 10^{-5} \AA^{-3}$. From Table I and the ideal gas equation $P=z \Omega k_{B} T$, we found this condition corresponds to a supersaturation $P / P_{\text {sat }}=5.8$. The formation free energy of the clusters at this supersaturation is shown in Fig. 5 (see curve $\mathrm{S}_{1}$ ). For $E=1.0 \times 10^{9} \mathrm{~V} / \mathrm{m}$, in order to keep the supersaturation $S=5.8$, we found from Table I and Eq. (10) that $z \Omega$ should be $0.23 \times 10^{-5} \AA^{-3}$. The curve $S_{2}$ in Fig. 5 shows simulation results under this condition. Finally, curve $S_{3}$ in Fig. 5 corresponds to the condition $E=1.0 \times 10^{9} \mathrm{~V} / \mathrm{m}$ and $z \Omega=0.31 \times 10^{-5} \AA^{-3}$ for which the pressure is set to be the same as that for curve $S_{1}$. 


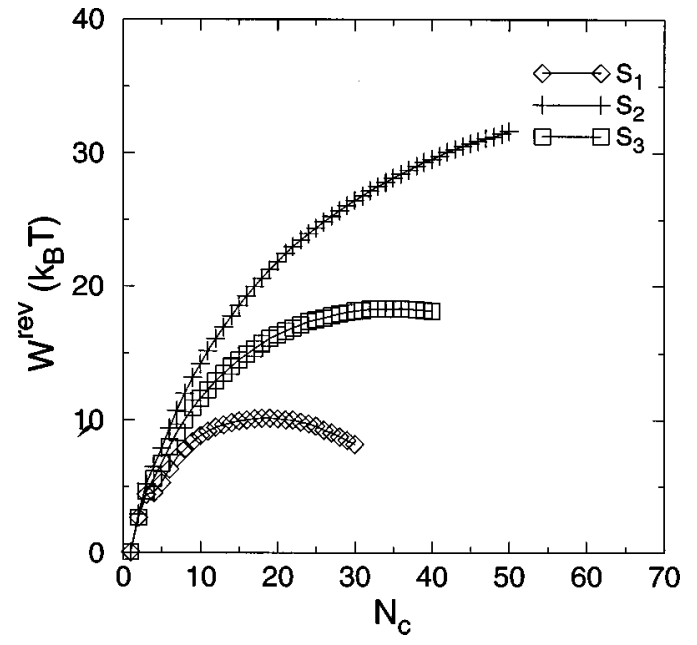

FIG. 5. The formation free energy of the SPC/E water clusters at $T$ $=323 \mathrm{~K}$. The curve $\mathrm{S}_{1}$ (open diamonds) is for $z \Omega=0.5 \times 10^{-5} \AA^{-3}$ and $E$ $=0 ; \mathrm{S}_{2}$ (crosses) is for $z \Omega=0.23 \times 10^{-5} \AA^{-3}$ and $E=1.0 \times 10^{9} \mathrm{~V} / \mathrm{m} ; \mathrm{S}_{3}$ (open squares) is for $z \Omega=0.31 \times 10^{-5} \AA^{-3}$ and $E=1.0 \times 10^{9} \mathrm{~V} / \mathrm{m}$. The same supersaturation is used for $S_{1}$ and $S_{2}$; the same vapor pressure is used for $S_{1}$ and $S_{3}$.

By comparing the three curves in Fig. 5, one can find that for both fixed supersaturation and fixed vapor pressure condition, the formation free energy tends to increase in the electric field. The formation free energy enhancement for the critical cluster is typically about $20 k_{B} T$ under the fixed supersaturation condition, and about $8 k_{B} T$ under the fixed pressure condition.

We also examined the shape of the clusters in the simulation. We found that when the size of the water cluster is small the cluster in a strong field is chain-like, a behavior similar to that found for a highly dipolar cluster in vacuum. ${ }^{31}$ As can be seen in Figs. 3 and 4, there exists a notable local maximum of $W^{\text {rev }}$ near $N_{c}=3$, particularly in the case of zero field. This maximum is due to the strong tendency of forming a stable hydrogen bonded network in the four cluster of water. ${ }^{18}$ However, in a strong field, the field tends to weaken this tendency. This is the reason why the local maximum around $N_{c}=3$ in Figs. 3 and 4 is smeared when the strongest field is applied. On the other hand, as the size of the water cluster becomes larger, the chain-like structure gradually disappears because of the strong tendency for water to form a compact hydrogen bonded network. A typical snapshot of the near-critical cluster under the electric field $E=1.0$ $\times 10^{9} \mathrm{~V} / \mathrm{m}$ at $T=323 \mathrm{~K}$ and $z \Omega=0.31 \times 10^{-5} \AA^{-3}$ is shown in Fig. 6. The size of the cluster is about 35 which is close to the critical cluster size at this state (see curve $S_{3}$ in Fig. 5). The elongated shape of this cluster can be clearly discerned.

\section{DISCUSSION AND CONCLUSION}

In this work, we employed GEMC and GCMC simulation methods to study the effects of a uniform electric field on the vapor-liquid coexistence (binodal) as well as the vapor-liquid nucleation of the SPC/E model water. We found that the field lowers the equilibrium vapor density and raises the equilibrium liquid density, and thus elevates the critical temperature. The main reason for the critical tem-

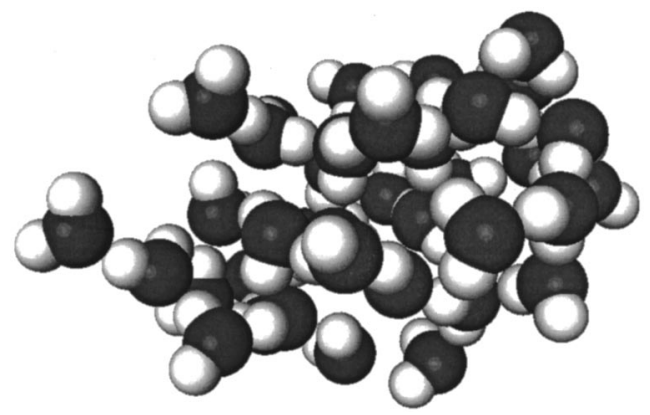

FIG. 6. A typical snapshot of a SPC/E water cluster under the external electric field of $E=1.0 \times 10^{9} \mathrm{~V} / \mathrm{m}$ at $T=323 \mathrm{~K}$ and $z \Omega=0.31 \times 10^{-5} \AA^{-3}$. The cluster size is 35 which is very close to the critical cluster size at this state (see curve $\mathrm{S}_{3}$ in Fig. 5).

perature rise is that the electric field effectively enhances the attractive interaction between molecules, as the dipole of the $\mathrm{SPC} / \mathrm{E}$ water molecules is forced to align along the direction of the field.

For the vapor-liquid nucleation, we found the condition of supersaturated vapor is essential to determine the external field effect. Under the fixed chemical potential condition, the field enhances the rate of nucleation. But under fixed supersaturation or vapor pressure condition, the field reduces the rate of nucleation. Among others, one important factor is the vapor density change in these states of supersaturated vapor. Under the fixed chemical potential condition, the vapor density $n_{v}$ increases with the field; under the fixed supersaturation condition $n_{v}$ actually decreases with the field. In most circumstances, the higher the vapor density is, the higher nucleation rate would be.

Under the fixed vapor pressure condition, the vapor density $n_{v}$ does not change with the field. In this case, the next important factor to the nucleation is likely the geometry shape of the cluster. Indeed, in the external field, the water cluster is elongated in the direction of the field ${ }^{28}$ (see Fig. 6) which gives rise to a larger surface-volume ratio of the cluster, and thus a larger surface contribution of the formation free energy. In this case, the formation free energy can be higher even though the molecules in the cluster have a stronger attractive interaction than those in zero field.

The conclusions of this work are in fact consistent with an earlier study of weakly dipolar Stockmayer fluids in a uniform field (part I). ${ }^{14}$ In that work, the i/v cluster theory of Reiss and co-workers was used to qualitatively calculate the formation free energy of dipolar clusters. The fact that these two different approaches predict qualitatively the same field effects on vapor-liquid nucleation is very encouraging.

Another conclusion of this paper is that for SPC/E model water, the electric field has neither an appreciable effect on the vapor-liquid coexistence nor on the vapor-liquid nucleation if the strength of the field is much less than $10^{9} \mathrm{~V} / \mathrm{m}$. This result is consistent with the experiment by Katz et al. ${ }^{3}$ that the field effects seen in the experiment are mainly due to the presence of ions in vapor, and that the rate of nucleation is independent of the field strength, provided the strength is less than $10^{4} \mathrm{~V} / \mathrm{m}$.

However, if a strong electric field with strength as high 
as $10^{9} \mathrm{~V} / \mathrm{m}$ was used in the laboratory, one would expect that the polarization should play an important role in this field because the strength of this field is comparable to the average strength of the local internal electric field in condensed water. The SPC/E model water is a rigid model of water, which cannot correctly account for the polarization effects under such a high field. It will be interesting to investigate the extent to which the polarization effects influence the rate of nucleation. To this end, polarizable model water should be used. ${ }^{21,32}$ Progress on this issue is underway and results will be presented in the next paper of this series.

The GCMC simulation method of Kusaka et al. offers a convenient way to calculate formation free energy of critical cluster with size, typically less than a hundred. Although approximations in this method (such as treating the supersaturated vapor as an ideal gas and neglecting vapor-cluster interaction) seem reasonable, a major limitation as mentioned in Sec. I is that it thus far can be applied only to molecule systems with a strong intermolecular interaction such as SPC/E water or a strongly dipolar Stockmayer fluid. $^{31}$ These systems have extremely low saturation pressure near the triple point. In passing, we note that a new method-the n/v-Stillinger cluster theory-is currently under development by Reiss and co-workers with the goal that the formation free energy of the physical cluster for any molecules can be quantitatively obtained. ${ }^{33}$

\section{ACKNOWLEDGMENTS}

The authors are grateful to Professor Z.-G. Wang, Professor A. Shchekin, Dr. I. Kusaka, Dr. K. Yasuoka, and Dr. K. Koga for helpful discussions. One of the authors (X.C.Z.) thanks the National Science Foundation for support of this work.

${ }^{1}$ H. Rabeony and P. Mirabel, J. Phys. Chem. 91, 1815 (1987).

${ }^{2}$ F. He and P. K. Hopke, J. Chem. Phys. 99, 9972 (1993).

${ }^{3}$ J. L. Katz, J. A. Fisk, and V. M. Chakarov, J. Chem. Phys. 101, 2309 (1994).

${ }^{4}$ R. Becker and W. Döring, Ann. Phys. (Leipzig) 24, 719 (1935).

${ }^{5}$ D. Kashchiev, Philos. Mag. 25, 459 (1972).

${ }^{6}$ J. O. Isard, Philos. Mag. 35, 817 (1977).
${ }^{7}$ K. J. Cheng, Phys. Lett. 106A, 403 (1984).

${ }^{8}$ V. B. Warshavsky and A. K. Shchekin (preprint).

${ }^{9}$ H. M. Ellerby, C. L. Weakliem, and H. Reiss, J. Chem. Phys. 95, 9209 (1991).

${ }^{10}$ H. M. Ellerby and H. Reiss, J. Chem. Phys. 97, 5766 (1992).

${ }^{11}$ C. L. Weakliem and H. Reiss, J. Chem. Phys. 99, 5374 (1993).

${ }^{12}$ C. L. Weakliem and H. Reiss, J. Chem. Phys. 101, 2398 (1994).

${ }^{13}$ K. J. Oh, X. C. Zeng, and H. Reiss, J. Chem. Phys. 107, 1242 (1997).

${ }^{14}$ K. J. Oh, G. T. Gao, and X. C. Zeng, J. Chem. Phys. 109, 8435 (1998).

${ }^{15}$ M. P. Allen and D. J. Tildesley, Computer Simulation of Liquids (Oxford University Press, Oxford, 1987).

${ }^{16}$ J. K. Lee, J. A. Barker, and F. F. Abraham, J. Chem. Phys. 58, 3166 (1973).

${ }^{17}$ H. J. C. Berendsen, J. R. Grigera, and T. P. Straatsma, J. Phys. Chem. 91, 6269 (1987)

${ }^{18}$ I. Kusaka, Z.-G. Wang, and J. H. Seinfeld, J. Chem. Phys. 108, 3416 (1998).

${ }^{19}$ A. Z. Panagiotopoulos, Mol. Phys. 61, 813 (1987).

${ }^{20}$ A. Z. Panagiotopoulos, N. Quirke, M. R. Stapleton, and D. J. Tildesley, Mol. Phys. 63, 527 (1988).

${ }^{21}$ S. B. Zhu, S. Singh, and G. W. Robinson, Adv. Chem. Phys. 85, 627 (1994).

${ }^{22}$ H. J. C. Berendsen, J. P. M. Postma, W. F. van Gunsteren, and J. Hermans, in Intermolecular Forces: Proceedings of the Fourteenth Jerusalem Symposium on Quantum Chemistry and Biochemistry, edited by Pullman (Reifel, Dordrecht, 1981), pp. 331-342.

${ }^{23}$ W. L. Jorgensen, J. Chandrasekhar, J. D. Madura, R. W. Impey, and M. L. Klein, J. Chem. Phys. 79, 926 (1983).

${ }^{24}$ Y. Guissani and B. Guillot, J. Chem. Phys. 98, 8221 (1993).

${ }^{25}$ J. Alejandre and D. J. Tildesley, J. Chem. Phys. 102, 4574 (1995).

${ }^{26}$ S. W. de Leeuw, J. W. Perram, and E. R. Smith, Proc. R. Soc. London, Ser. A 373, 27 (1980)

${ }^{27}$ E. Schmidt, Properties of Water and Steam in SI-Unit (Springer, Berlin, 1969).

${ }^{28}$ M. J. Stevens and G. S. Grest, Phys. Rev. E 51, 5976 (1995).

${ }^{29}$ D. Chandler, Introduction to Modern Statistical Mechanics (Oxford University Press, New York, 1987).

${ }^{30}$ C. G. Gray and K. E. Gubbins, Theory of Molecular Fluids (Clarendon, Oxford, 1984)

${ }^{31}$ P. R. ten Wolde, D. W. Oxtoby, and D. Frenkel, Phys. Rev. Lett. 81, 3695 (1998).

${ }^{32}$ For the latest development on the polarizable water model see, for example, S. W. Rick, S. J. Stuart, and B. J. Berne, J. Chem. Phys. 101, 6141 (1994); D. N. Bernardo, Y. Ding, K. Krogh-Jespersen, and R. M. Levy, J. Phys. Chem. 98, 4180 (1994); J. Brodholt, M. Sampoli, and R. Vallauri, Mol. Phys. 86, 149 (1995); P. T. Kusalik, F. Liden, and I. M. Svishchev, J. Chem. Phys. 103, 10169 (1995); A. A. Chialvo and P. T. Cummings, ibid. 105, 8274 (1996).

${ }^{33}$ B. Senger, P. Schaaf, D. S. Corti, R. Bowles, D. Pointu, J.-C. Voegel, and H. Reiss, J. Chem. Phys. (in press) 